\title{
Flocked nasal swab versus nasopharyngeal aspirate for detection of respiratory tract viruses in immunocompromised adults: a matched comparative study
}

\author{
Lars Öhrmalm", Michelle Wong ${ }^{1}$, Maria Rotzén-Östlund², Oscar Norbeck ${ }^{1}$, Kristina Broliden ${ }^{1}$, Thomas Tolfvenstam ${ }^{1}$
}

\begin{abstract}
Background: Several studies have compared nasal swabs to the more invasive nasopharyngeal aspirate (NPA) for detection of respiratory viruses. Mostly, the comparisons have been performed on immunocompetent children with upper respiratory tract symptoms. The results range from a relatively poor sensitivity for the swabs to an even higher sensitivity than for the NPA. We aimed to investigate the sensitivity of a flocked nasal swab (fNS) on immunocompromised adults with febrile neutropenia.
\end{abstract}

Methods: During 16 months, adults with a hematological disorder presenting with febrile neutropenia were enrolled in the study. Paired samples of the fNS and NPA were collected in the outer part of the nasal cavity and the nasopharynx, respectively. The samples were analyzed regarding a panel of 15 respiratory viruses by means of quantitative polymerase chain reaction. Furthermore, as an indirect measure of cell yield by either method, the copy number of the human beta actin gene was also determined. Cohen's kappa was calculated as a measure of agreement of the results obtained from either method. Wilcoxon signed-rank test was used for comparison of cell yield.

Results: A total of 98 paired samples from a total of 89 patients were collected. Twenty of the pairs had virus detected in at least one of the specimens; 11 in both, 7 in NPA only, and 2 in fNS only. For the fNS, the overall sensitivity for any virus and for rhinovirus only was $65 \%$ and $78 \%$, respectively. NPA was significantly superior to the fNS in collecting epithelial cells.

Conclusion: We found the overall sensitivity of $65 \%$ to be too low to replace NPA with this sampling technique in this patient category.

\section{Background}

A number of studies have compared different sampling techniques for detection of viruses in the upper respiratory tract in immunocompetent children [1-14]. The advantages of using a swab in the nares compared to nasopharyngeal aspirate (NPA) are for the patient less discomfort and more rapid sampling procedure. For the medical personnel there is a time gain. Finally, the swab goes with a lower cost than does the NPA. Respiratory

\footnotetext{
* Correspondence: lars.ohrmalm@ki.se

'Department of Medicine, Solna, Infectious Disease Unit, Center for Molecular Medicine, Karolinska Institutet, Karolinska University Hospital, SE17176 Stockholm, Sweden

Full list of author information is available at the end of the article
}

viruses are common findings in children [15] and adults with hematological malignancies and have been recognized as a potential cause of pneumonia and death [16]. Thus, the sampling frequency for detection of respiratory viruses in this patient category is expected to increase. However, most of the studies comparing sampling techniques are performed on children and, as to our knowledge; to date no study has been performed on immunocompromised individuals which could have a reduced local immunological and inflammatory response, making a direct clinical application of to date achieved results and conclusions impossible. As the swab has been suggested to be comparable with 
NPA $[1,4,9]$, the primary objective of this study was to determine the sensitivity of detecting respiratory viruses in immunocompromised adults using a flocked nasal swab (fNS) in the outer part of the nose cavity compared to NPA.

\section{Methods}

\section{Participants}

Between January 2008 and May 2009 adults with any hematological disorder presenting at the Karolinska University Hospital, Stockholm, for febrile neutropenia (auricular temperature $>38.0^{\circ} \mathrm{C}$ twice or $\geq 38.5^{\circ} \mathrm{C}$ at one occasion, and an absolute neutrophil count $\leq 500$ cells $/ \mathrm{mm}^{3}$ ) were asked to participate in this study. The patients were allowed to participate more than once provided that an afebrile period of at least three weeks separated the episodes of febrile neutropenia. At admission, the patients received empirically administrated broad-spectrum antibiotics; ceftazidime or piperacillin-tazobactam.

\section{Collection and storage of material}

The collection was made within 72 hours from onset of fever. The fNS with a nylon fiber tip (COPAN, art. no. CP552C) was inserted at least $20 \mathrm{~mm}$ and rotated inside each nostril. Then, the NPA was obtained by insertion of a sterile catheter (no. 8, Mediplast, Sweden) into the posterior nasopharynx and pulled back while applying gentle suction. Finally, 2-3 mL of sodium chloride was sucked into the trap. Both specimens were obtained without instillation of any solution into the nostrils. The specimens were stored without any medium in room temperature and transported to the laboratory within six hours. The NPA was stored in its collection tube in minus $80^{\circ} \mathrm{C}$. The fiber tip of the fNS was put in $500 \mu \mathrm{L}$ RPMI 1640 (Sigma-Aldrich, St. Louis, MO) and shaken for $30 \mathrm{~min}-$ utes. The suspension was stored in minus $80^{\circ} \mathrm{C}$.

\section{Detection methods}

A total of $400 \mu \mathrm{L}$ of each sample was extracted and then analyzed regarding presence of nucleic acids from adenovirus, bocavirus, coronavirus, enterovirus, influenzavirus $A+B$, metapneumovirus, parainfluenzavirus 1-3, rhinovirus, and RS-virus. The extraction method and the quantitative polymerase chain reaction (qPCR) assay are previously described [10]. A TaqMan qPCR assay was developed based on amplification of the human beta actin gene (ACTB) (forward primer 5'-GCGCGGCTACAGCTTCA-3' [50 nM], reverse primer 5'-GCGCGGCTACAGCTTCA-3' [900 nM], probe 6FAM-CACCACGGCCGAGC-MGB [150 nM]). The assay was performed on an ABI 7500 Real-Time PCR System (Applied Biosystems). The PCR assay was carried out in a total $50-\mu \mathrm{L}$ reaction mixture containing
$25 \mu \mathrm{L}$ of TaqMan Universal PCR Master Mix (Applied Biosystems) and $5 \mu \mathrm{L}$ of template, leaving $20 \mu \mathrm{L}$ to the primers and probe. The PCR program included 1 cycle at $50^{\circ} \mathrm{C}$ for $2 \mathrm{~min}, 1$ cycle at $95^{\circ} \mathrm{C}$ for $10 \mathrm{~min}$, followed by 40 cycles consisting of $15 \mathrm{sec}$ at $95^{\circ} \mathrm{C}$ and $60 \mathrm{sec}$ at $60^{\circ} \mathrm{C}$. The sensitivity of the assay was 3 copies per reaction, as determined by repeated testing of in-house cloned plasmids.

\section{Ethics}

After giving written informed consent the patients were enrolled. The study was approved by The Regional Ethical Review Board in Stockholm that handles applications for research at Karolinska University Hospital.

\section{Statistical methods}

Positivity by either method was used as gold standard for presence of viruses. Cohen's kappa was calculated as a measure of agreement of the results obtained from either method [17]. Wilcoxon signed-rank test and Pearson's correlation coefficient were used when appropriate. Age is presented as means $\pm \mathrm{SD}$, whereas neutrophil count is presented as median followed by range. A p-value $<0.05$ was considered significant. InStat 3.05 and Prism 5.00 for Windows were used.

\section{Results}

A total of 98 episodes of febrile neutropenia occurring in 89 patients were included in the study. Based on episodes, the mean age was $55 \pm 15$ years ( 43 females, $57 \pm$ 13 years; 55 males, $53 \pm 16$ years) and the median neutrophil count $<100$ cells $/ \mathrm{mm}^{3}(<100-500)$. As underlying hematological disorder, acute leukemia and myelodysplastic syndrome predominated $(n=42,43 \%)$ followed by non-Hodgking lymphoma $(\mathrm{n}=33,34 \%)$ and myeloma $(\mathrm{n}=13,13 \%)$. The remaining diagnoses were chronic lymphocytic leukemia $(n=6,6 \%)$ and Hodgking's lymphoma $(\mathrm{n}=4,4 \%)$. In total, $49(50 \%)$ of the patients were on antiviral prophylaxis with aciclovir. The patients were sampled within one day from admittance $(\mathrm{n}=54,55 \%)$, but due to practical obstacles, in 32 (33\%) and $9(9 \%)$ of the cases, the samples were collected on the second and third day after admittance, respectively. In total, 98 paired samples collected with fNS and NPA respectively, were obtained. A total of 20 of the pairs had virus detected in one or both of the specimens; 7 exclusively in NPA and 2 exclusively in fNS (Table 1). The sensitivity for the fNS in detection of any virus was $65 \%(95 \% \mathrm{CI}=41-85)$ and for rhinovirus $78 \%(95 \% \mathrm{CI}=40-97)$. The positivity rates for any virus for the NPA and fNS were 18.4\% (18 of 98) and 13.3\% (13 of 98), respectively, showing a "substantial" agreement (for rhinovirus an "almost perfect" agreement) according to Landis and Koch's scale [17]. 
Table 1 Detection of viruses in NPA and fNS specimens compared with total viral findings by either method

\begin{tabular}{|c|c|c|c|c|c|c|c|}
\hline \multirow[t]{2}{*}{ Virus } & \multicolumn{3}{|c|}{$\begin{array}{l}\text { No. of samples in which } \\
\text { virus was detected by: }\end{array}$} & \multirow[t]{2}{*}{$\begin{array}{c}\text { Total } \\
\text { no. }\end{array}$} & \multirow[t]{2}{*}{$\begin{array}{l}\text { Sensitivity }{ }^{a} \text { for } \\
\text { fNS (\%) }\end{array}$} & \multirow[t]{2}{*}{$\begin{array}{l}\text { Mean difference }{ }^{b} \text { in } \mathrm{Ct} \\
\text { values (range) }\end{array}$} & \multirow[t]{2}{*}{$\begin{array}{c}\text { Agreement }{ }^{c} \text { between NPA and } \\
\text { fNS }\left(95 \% \mathrm{Cl}^{d}\right)\end{array}$} \\
\hline & $\begin{array}{c}\text { Both } \\
\text { methods }\end{array}$ & $\begin{array}{l}\text { NPA } \\
\text { only }\end{array}$ & $\begin{array}{l}\text { fNS } \\
\text { only }\end{array}$ & & & & \\
\hline Rhinovirus & 7 & 2 & 0 & 9 & 78 & $3.2(-2.0-6.5)$ & $0.86(0.68-1.05)$ \\
\hline RSV A & 1 & 1 & 1 & 3 & & 5.8 & \\
\hline RSV B & 2 & 0 & 0 & 2 & & $2.2(5.6-9.9)$ & \\
\hline Influenza A & 0 & 1 & 0 & 1 & & N/A & \\
\hline Influenza B & 1 & 1 & 0 & 2 & & 15.5 & \\
\hline Bocavirus & 0 & 1 & 0 & 1 & & N/A & \\
\hline Enterovirus & 0 & 1 & 0 & 1 & & N/A & \\
\hline Metapneumovirus & 0 & 0 & 1 & 1 & & N/A & \\
\hline Any virus & 11 & 7 & 2 & 20 & 65 & $5.1(-2.0-15.5)$ & $0.66(0.44-0.87)$ \\
\hline
\end{tabular}

NOTE. NPA, nasopharyngeal aspirate; fNS, flocked nasal swab; $\mathrm{Ct}$, cycle of threshold; $\mathrm{Cl}$, confidence interval.

a Percentage of positive samples from fNS in relation to the total number of pairs with positive results from any of specimens.

b The $\mathrm{Ct}$ value for the flocked nasal swab minus the corresponding $\mathrm{Ct}$ value for NPA.

' Cohen's kappa: >0.8, almost perfect agreement; 0.6-0.8, substantial agreement; 0.4-0.6, moderate agreement; <0.4, poor agreement.

${ }^{\mathrm{d}} \mathrm{Cl}$, confidence interval.

The amount of cells yielded by NPA approximated by ACTB copy numbers was significantly larger than by $\mathrm{fNS}$ with a mean difference of $4.2 \times 10^{5}$ cells $/ \mathrm{mL}(p=$ .001). In pairs with virus detected in both methods, the Ct values were lower (thus viral load higher) for NPA than for fNS. We plotted the difference in cell count between fNS and NPA against the difference in $\mathrm{Ct}$ values between the methods for the positive pairs. Although not statistically significant, the correlation indicate a possible association between a high cell yield and low $\mathrm{Ct}$ value $(\mathrm{r}=-0.46, p=.15)$.

\section{Discussion}

We compared the efficacy to collect specimen for viral detection between fNS and NPA. According to Landis and Koch's [17] scale there was a substantial agreement between the methods, to NPA's favor, and NPA was significantly more efficient collecting epithelial material approximated by quantification of ACTB. Even if removal of the cellular component of NPA does not interfere with quantification of some respiratory viruses [18], a higher cell count might indicate a higher yield of specimen. Even though not statistically significant, the $\mathrm{Ct}$ value negatively correlated to the number of cells collected, and thus, the finding of NPA being superior to the fNS in collecting cells invite us to speculate that the fNS sometimes may collect inadequate amount of specimen in order to reach the PCR method's detection limit. Furthermore, several reports have suggested that sensitivity increases when the nasopharynx is sampled instead of the nasal cavity $[2,5,11,14]$.

Thus, the difference in sensitivity for the fNS and NPA could have several reasons; biological and technical. One obvious biological reason is the fact that two different anatomical sites are investigated; one technical explanation could be that suction is superior scratching for collecting adequate amount material. The production of mucus that transports the viral nucleic acids in an anterior direction to the fNS's sampling site may be an important parameter. In this patient category, the lack of a competent immune response could reduce the mucus-production. Taking the above into account, the depth of the sampling with the fNS probably plays an important role. However, a deeper penetration could cause even more discomfort than does the NPA.

In contrast to our findings, several groups have reached good sensitivity using the fNS in the outer part of the nose and concluded it comparable to NPA $[1,4,9]$. Sung et al who used an insertion depth of only 1-1.5 cm summarized the fNS an even better option when using PCR.

This study has several limitations. The time interval between fever onset and sampling varied between the patients, and they did not necessarily have respiratory tract symptoms. Furthermore, the patients suffered from different underlying diseases. However, this reflects the clinical reality and as both sampling methods were used at the same time point in the same patient, the comparison is still valid. The foremost important limitation is the low number of positive samples on which the calculation of sensitivity is based. However, the upper limit in the $95 \%$ confidence interval for the overall sensitivity was $85 \%$ which is still a questionable value for replacing an established method.

In summary, the NPA was superior to the fNS in collecting epithelial cells. Furthermore, we also found lower sensitivity for the fNS than for NPA in detecting respiratory viruses in these immunocompromised adults. 
For detection of rhinovirus it was slightly better, but still not appropriate, according to our judgment, for clinical use in this patient category. With an insertion depth comparable to what other groups have used we found lower sensitivity for the fNS than previous investigators. Less mucus production and consequently less anterior transport of viral nucleic acid in immunocompromised individuals may explain this finding.

\section{Conclusions}

On the basis of our findings, fNS sampling of the nares for viral detection in immunocompromised individuals cannot be recommended until further large studies have revealed more promising results.

\section{Acknowledgements}

We thank all involved patients. We are indebted to the nurses and study nurses at the Hematology Center and the Department of Emergency, Karolinska University Hospital, for their dedication to the study. We acknowledge the staff at the Department of Clinical Microbiology at Karolinska University Hospital. Grants were received from the Stockholm City Council/Karolinska Institutet and from the Swedish Children's Cancer Foundation.

\section{Author details}

${ }^{1}$ Department of Medicine, Solna, Infectious Disease Unit, Center for Molecular Medicine, Karolinska Institutet, Karolinska University Hospital, SE17176 Stockholm, Sweden. ${ }^{2}$ Department of Clinical Microbiology, Karolinska University Hospital and Department of Microbiology, Tumor and Cell biology, Karolinska Institutet, Stockholm, Sweden.

\section{Authors' contributions}

All authors have been active in the interpretation of data, the writing of the manuscript and the process of its internal revision as well as the final approval for submission. Furthermore, LÖ has contributed to the study regarding overall design, inclusion of a portion of the patients, acquisition of clinical data, and analysis of data. MW has contributed with laboratory design, compilation, and analysis of data. MRÖ contributed to the study regarding laboratory design, performance of the laboratory work and interpretation of raw data. ON has contributed to the overall design, inclusion of a portion of the patients, and analysis (including statistics) of data. KB was involved in the overall design, and analysis of data. TT contributed to the study regarding initiation and overall design, inclusion of a portion of the patients, acquisition of clinical data and analysis of data.

\section{Competing interests}

The authors declare that they have no competing interests.

Received: 8 September 2010 Accepted: 26 November 2010 Published: 26 November 2010

\section{References}

1. Sung RY, Chan PK, Choi KC, Li AM, Nelson EA: A comparative study of nasopharyngeal aspirate and nasal swab specimens for the diagnosis of acute viral respiratory infection. Hong Kong Med J 2009, 15(3 Suppl 4):24-27.

2. Heikkinen T, Salmi AA, Ruuskanen O: Comparative study of nasopharyngeal aspirate and nasal swab specimens for detection of influenza. BMJ 2001, 322(7279):138.

3. Lambert SB, Whiley DM, O'Neill NT, Andrews EC, Canavan FM, Bletchly C, Siebert DJ, Sloots TP, Nissen MD: Comparing nose-throat swabs and nasopharyngeal aspirates collected from children with symptoms for respiratory virus identification using real-time polymerase chain reaction. Pediatrics 2008, 122(3):e615-620.
4. Abu-Diab A, Azzeh M, Ghneim R, Zoughbi M, Turkuman S, Rishmawi N, Issa $A E$, Siriani I, Dauodi $R$, Kattan $R$, et al: Comparison between pernasal flocked swabs and nasopharyngeal aspirates for detection of common respiratory viruses in samples from children. J Clin Microbiol 2008, 46(7):2414-2417.

5. Daley P, Castriciano S, Chernesky M, Smieja M: Comparison of flocked and rayon swabs for collection of respiratory epithelial cells from uninfected volunteers and symptomatic patients. J Clin Microbiol 2006, 44(6):2265-2267.

6. Spyridaki IS, Christodoulou I, de Beer L, Hovland V, Kurowski M, OlszewskaZiaber A, Carlsen KH, Lodrup-Carlsen K, van Drunen CM, Kowalski ML, et a: Comparison of four nasal sampling methods for the detection of viral pathogens by RT-PCR-A GA(2)LEN project. J Virol Methods 2009, 156(12):102-106.

7. Stensballe LG, Trautner S, Kofoed PE, Nante E, Hedegaard K, Jensen IP, Aaby P: Comparison of nasopharyngeal aspirate and nasal swab specimens for detection of respiratory syncytial virus in different settings in a developing country. Trop Med Int Health 2002, 7(4):317-321.

8. Ahluwalia G, Embree J, McNicol P, Law B, Hammond GW: Comparison of nasopharyngeal aspirate and nasopharyngeal swab specimens for respiratory syncytial virus diagnosis by cell culture, indirect immunofluorescence assay, and enzyme-linked immunosorbent assay. Clin Microbiol 1987, 25(5):763-767.

9. Walsh P, Overmyer CL, Pham K, Michaelson S, Gofman L, DeSalvia L, Tran T, Gonzalez D, Pusavat J, Feola M, et al: Comparison of respiratory virus detection rates for infants and toddlers by use of flocked swabs, saline aspirates, and saline aspirates mixed in universal transport medium for room temperature storage and shipping. I Clin Microbiol 2008, 46(7):2374-2376

10. Tiveljung-Lindell A, Rotzen-Ostlund M, Gupta S, Ullstrand R, Grillner L, Zweygberg-Wirgart B, Allander T: Development and implementation of a molecular diagnostic platform for daily rapid detection of 15 respiratory viruses. J Med Virol 2009, 81(1):167-175.

11. Heikkinen T, Marttila J, Salmi AA, Ruuskanen O: Nasal swab versus nasopharyngeal aspirate for isolation of respiratory viruses. J Clin Microbiol 2002, 40(11):4337-4339.

12. Frayha H, Castriciano S, Mahony J, Chernesky M: Nasopharyngeal swabs and nasopharyngeal aspirates equally effective for the diagnosis of viral respiratory disease in hospitalized children. J Clin Microbiol 1989, 27(6):1387-1389.

13. Ipp M, Carson S, Petric M, Parkin PC: Rapid painless diagnosis of viral respiratory infection. Arch Dis Child 2002, 86(5):372-373.

14. Macfarlane P, Denham J, Assous J, Hughes C: RSV testing in bronchiolitis: which nasal sampling method is best? Arch Dis Child 2005, 90(6):634-635.

15. Lindblom A, Bhadri V, Soderhall S, Ohrmalm L, Wong M, Norbeck O, Lindau C, Rotzen-Ostlund M, Allander T, Catchpoole D, et al: Respiratory viruses, a common microbiological finding in neutropenic children with fever. J Clin Virol 47(3):234-237.

16. Chemaly RF, Ghosh S, Bodey GP, Rohatgi N, Safdar A, Keating MJ, Champlin RE, Aguilera EA, Tarrand JJ, Raad II: Respiratory viral infections in adults with hematologic malignancies and human stem cell transplantation recipients: a retrospective study at a major cancer center. Medicine (Baltimore) 2006, 85(5):278-287.

17. Landis JR, Koch GG: The measurement of observer agreement for categorical data. Biometrics 1977, 33(1):159-174.

18. Semple MG, Booth JA, Ebrahimi B: Most human metapneumovirus and human respiratory syncytial virus in infant nasal secretions is cell free. $J$ Clin Virol 2007, 40(3):241-244

\section{Pre-publication history}

The pre-publication history for this paper can be accessed here: http://www.biomedcentral.com/1471-2334/10/340/prepub

\section{doi:10.1186/1471-2334-10-340}

Cite this article as: Öhrmalm et al: Flocked nasal swab versus nasopharyngeal aspirate for detection of respiratory tract viruses in immunocompromised adults: a matched comparative study. BMC Infectious Diseases 2010 10:340. 